\title{
Endoplasmic Reticulum and Lysosomal Quality Control of Four Nonsense Mutants of Iduronate 2-Sulfatase Linked to Hunter's Syndrome
}

\author{
Alessandro Marazza, ${ }^{1-3}$ Carmela Galli, ${ }^{1,2}$ Elisa Fasana, ${ }^{1,2}$ Jacopo Sgrignani, ${ }^{1,2}$ Patricie Burda, ${ }^{4}$ \\ Enrico M.A. Fassi ${ }^{1,2}$ Matthias Baumgartner, ${ }^{4}$ Andrea Cavalli, ${ }^{1,2,5}$ and Maurizio Molinari ${ }^{1,2,6}$
}

\begin{abstract}
Hunter's syndrome (mucopolysaccharidosis type II) is a rare X-linked lysosomal storage disorder caused by mutations in the iduronate-2-sulfatase (IDS) gene. Motivated by the case of a child affected by this syndrome, we compared the intracellular fate of wild-type IDS (IDS $\mathrm{WT}_{\mathrm{W}}$ ) and four nonsense mutations of IDS (IDS $\mathrm{L} 482 \mathrm{X}_{\text {, }}$ IDS $_{\mathrm{Y} 452 \mathrm{X}}, \mathrm{IDS}_{\mathrm{R} 443 \mathrm{X}}$, and $\left.\mathrm{IDS}_{\mathrm{W} 337 \mathrm{X}}\right)$ generating progressively shorter forms of IDS associated with mild to severe forms of the disease. Our analyses revealed formylation of all forms of IDS at cysteine 84, which is a prerequisite for enzymatic activity. After formylation, IDS $_{\mathrm{WT}}$ was transported within lysosomes, where it was processed in the mature form of the enzyme. The length of disease-causing deletions correlated with gravity of the folding and transport phenotype, which was anticipated by molecular dynamics analyses. The shortest form of IDS, IDS $\mathrm{W}_{\mathrm{W} 37 \mathrm{X}}$, was retained in the endoplasmic reticulum (ER) and degraded by the ubiquitin-proteasome system. IDS $\mathrm{R}_{\mathrm{R} 43 \mathrm{X}}, \mathrm{IDS}_{\mathrm{Y} 452 \mathrm{X}}$, and $\mathrm{IDS}_{\mathrm{L} 482 \mathrm{X}}$ passed ER quality control and were transported to the lysosomes, but failed lysosomal quality control, resulting in their rapid clearance and in loss-of-function phenotype. Failure of ER quality control inspection is an established cause of loss of function observed in protein misfolding diseases. Our data reveal that fulfillment of ER requirements might not be sufficient, highlight lysosomal quality control as the distal station to control lysosomal enzymes fitness and pave the way for alternative therapeutic interventions.
\end{abstract}

Keywords: endoplasmic reticulum, formylation, glycosaminoglycans, Hunter's syndrome, iduronate-2-sulfatase, nonsense mutations, lysosomal storage diseases, lysosome, molecular dynamics, mucopolysaccharidosis type II

\section{Introduction}

$\mathbf{L}$ YSOSOMAL STORAGE DISORDERS (LSDs) are rare inherited progressive diseases characterized by impaired lysosomal activity with abnormal accumulation of macromolecules in the lumen of lysosomes (Marques and Saftig, 2019). Mucopolysaccharidosis type II (MPS II, Hunter's syndrome) (Hunter, 1917) is a rare X-linked recessive LSD characterized by mutations of the gene encoding for iduronate-2-sulfatase (IDS) (Bach et al., 1973; Wraith et al., 2008; Stenson et al., 2014). IDS deficiency leads to accumulation of glycosaminoglycans within the lysosomes of cells and tissues throughout the body (Muenzer et al., 2009). MPS II patients exhibit multiorgan symptoms, including progressive deafness, skeletal deformities, carpal tunnel syndrome, hepatosplenomegaly, frequent respiratory infections, cognitive impairment, and hydrocephalus (Muenzer et al., 2009; Whiteman and Kimura, 2017). The IDS gene encodes a pre-propolypeptide of 550 residues. During endoplasmic reticulum (ER) translocation, side chains of seven asparagine residues are $N$-glycosylated, the cysteine 84 is modified in formylglycine (oxoalanine) as key catalytic residue in the IDS active site (Schmidt et al., 1995), and two intramolecular disulfide bonds are formed (Fig. 1A) (Demydchuk et al., 2017). The native polypeptide is transported to the lysosomes, where it is proteolytically activated on removal of part of the $N$-terminus and cleavage of the SD2 regulatory peptide that remains noncovalently associated

\footnotetext{
${ }^{1}$ Università della Svizzera italiana, Lugano, Switzerland.

${ }^{2}$ Institute for Research in Biomedicine, Bellinzona, Switzerland.

${ }^{3}$ Graduate School for Cellular and Biomedical Sciences, University of Bern, Bern, Switzerland.

${ }^{4}$ Abteilung für Stoffwechselkrankheiten, Kinderspital Zürich, Zurich, Switzerland.

${ }^{5}$ Swiss Institute of Bioinformatics, Lausanne, Switzerland.

${ }^{6}$ École Polytechnique Fédérale de Lausanne, Lausanne, Switzerland.
} 
A

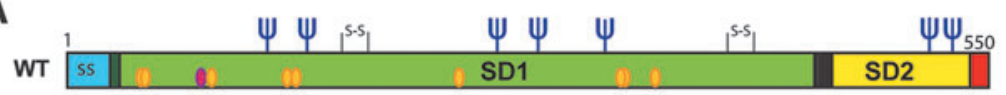

FL
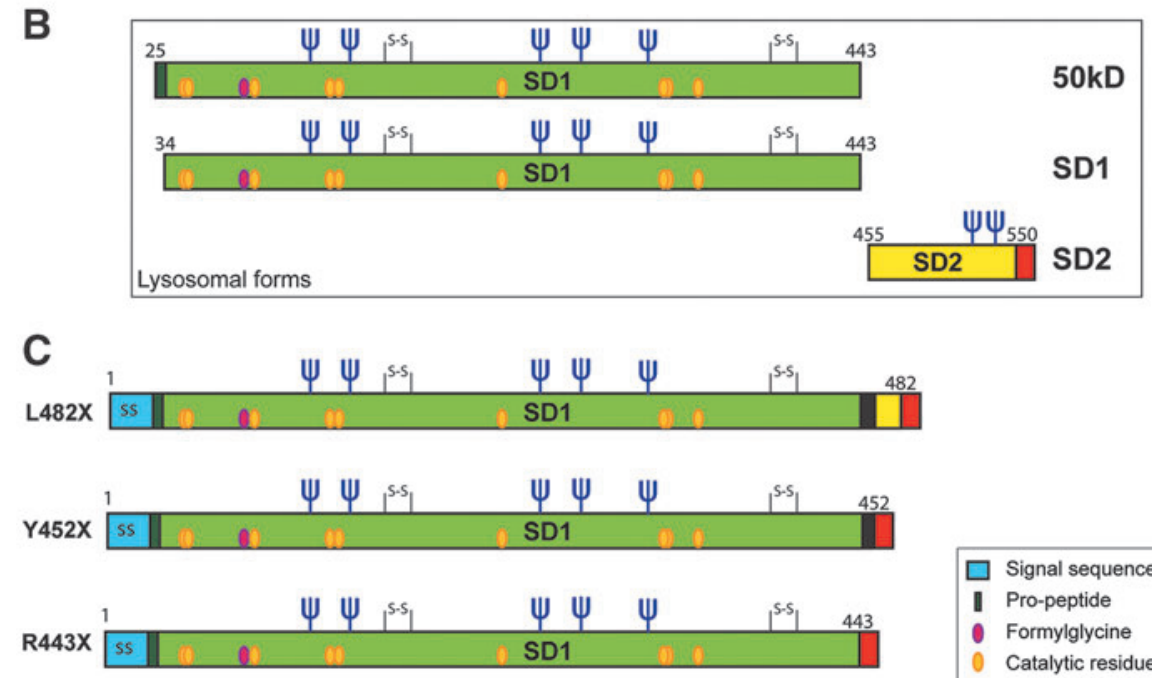

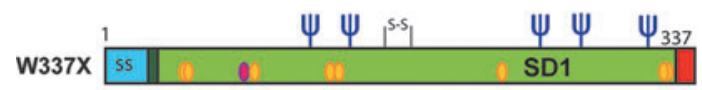
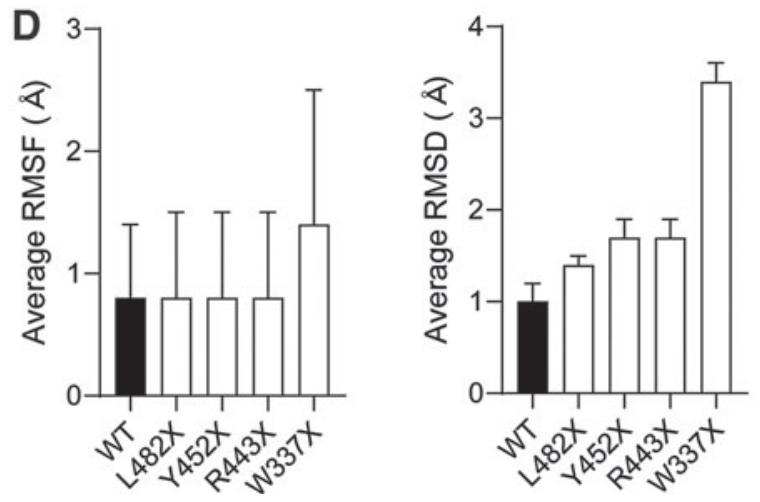

FIG. 1. IDS variants and their predicted stability. (A) Full-length (FL) IDS $_{\mathrm{WT}}$. The position of ss, propeptide, subdomains, catalytic residues, $N$-glycans, and disulfide bonds, and number of residues is given. The proteins are tagged at their C-termini. (B) Products of proteolytic maturation. (C) Disease-causing, truncated forms of IDS analyzed in this study. (D) Molecular dynamics simulations. RMSF correlate with protein stability and RMSD express deviation from the reference IDS $_{\mathrm{WT}}$ crystal structure. IDS, iduronate 2-sulfatase; IDS $_{\mathrm{WT}}$, wild type IDS; RMSD, root mean square deviation; RMSF, root mean square fluctuations; ss, signal sequence. with the catalytic SD1 domain (Fig. 1B) (Froissart et al., 1995; Demydchuk et al., 2017). More than 500 IDS mutations have been reported, but little is known about consequences thereof on IDS biogenesis and lysosomal transport (Demydchuk et al., 2017). Analysis of few MPS II-causing IDS mutants recently led to ascribe the associated loss-offunction disorder to mutant's clearance by ER-associated degradation (ERAD) and to propose ERAD modulators as targets for therapeutic intervention (Osaki et al., 2018, 2019). Motivated by the request of a family to study the IDS $_{\mathrm{W} 337 \mathrm{X}}$ nonsense mutant, we compared its intracellular fate and the intracellular fate of three other MPS II-causing nonsense mutants (i.e., IDS $_{\mathrm{R} 443 \mathrm{X}}, \mathrm{IDS}_{\mathrm{Y} 452 \mathrm{X}}$, and $\mathrm{IDS}_{\mathrm{L} 482 \mathrm{X}}$ ) with that of the wild-type IDS (IDS $\mathrm{WT}_{\mathrm{WT}}$ ) protein. The clinical correlation between patients with the same mutation is imperfect. However, according to the literature, the truncation at residue 337 invariably correlates with severe MPS II (Sukegawa et al., 1995; Keeratichamroen et al., 2008; Kosuga et al., 2016), whereas truncations at residues 443, 452 , and 482 are characterized by intermediate to mild forms of the loss-of-function disease (Sukegawa et al., 1995; Froissart et al., 1998; Vafiadaki et al., 1998; Filocamo et al., 2001; Keeratichamroen et al., 2008; Sohn et al., 2012; Brusius-Facchin et al., 2014; Kosuga et al., 2016). In this study, we performed molecular dynamic (MD) simulations that revealed structural instability only for IDS $_{\mathrm{W} 337 \mathrm{X}}$. These predictions were eventually supported by in cellulo analyses showing that IDS $_{\mathrm{W} 337 \mathrm{X}}$ does not pass ER quality control and is cleared by ERAD. IDS $\mathrm{R}_{443 \mathrm{X}}, \mathrm{IDS}_{\mathrm{Y} 452 \mathrm{X}}, \mathrm{IDS}_{\mathrm{L} 482 \mathrm{X}}$, and IDS $_{\mathrm{WT}}$ pass ER quality control and are transported within lysosomes. However, in contrast to $\mathrm{IDS}_{\mathrm{WT}}$, which is processed into the enzymatically active form, IDS $_{\mathrm{R} 443 \mathrm{X}}$, ID$\mathrm{S}_{\mathrm{Y} 452 \mathrm{X}}$, and $\mathrm{IDS}_{\mathrm{L} 482 \mathrm{X}}$ are rapidly degraded. All in all, in addition to available literature indicating the failure to pass ER quality control and the subsequent intervention of the ubiquitin proteasome system as causative of loss-of-function phenotypes [(Ulloa-Aguirre et al., 2004) and (Osaki et al., 2018, 2019) for the specific case of MPS II], our data involve the lysosomal compartment as a major site of clearance of structurally aberrant gene products linked to LSDs. 


\section{Materials and Methods}

IDS plasmids, primers, antibodies, and inhibitors

IDS $_{\mathrm{WT}}$ and IDS $_{\mathrm{W} 337 \mathrm{X}}$ constructs were ordered by GenScript (Piscataway). IDS ID443X $_{\text {IDS }}$ ID452X, and $\operatorname{IDS}_{\mathrm{L} 482 \mathrm{X}}$ were generated in our laboratory, amplifying pcDNA3.1 containing the IDS $_{\mathrm{WT}}$ sequence by PCR using $5^{\prime}$-GATCACA CCGGTACCCCACCATGCCGCCA-3' as forward primer and, as reverse primers, 5'-ACCTTCCTGAAGCATTTTG CGGCCGCCCCACACTAG-3' for IDS $_{\mathrm{R} 443 \mathrm{X}}, 5^{\prime}$-ACTT GGAAGAGGATCCGGCGGCCGCCCCACACTAG-3' for IDS $_{\mathrm{Y} 452 \mathrm{X}}$, and 5'-ATTCTGACAAGCCGAGTGCGGCCG CCCCACACTAG-3' for IDS $_{\mathrm{L} 482 \mathrm{X}}$. HA-tag antibodies are from Sigma-Aldrich (Buchs, Switzerland), anti-IDS from R\&D Systems (Minneapolis). PS341 (Millenium Pharmaceuticals), and BafA1 (Calbiochem and Sigma Aldrich, Buchs, Switzerland) were used at final concentration of $10 \mu \mathrm{M}$ and $50-100 \mathrm{nM}$, respectively.

\section{Cell lines and transient transfection}

HEK and mouse embryonic fibroblasts (MEF) were cultured in $4.5 \mathrm{~g} / \mathrm{L}$ D glucose D-MEM (Thermo Fisher Scientific, Waltham) containing $10 \%$ fetal bovine serum. Cells were seeded in $6 \mathrm{~cm}$ culture dishes/12-well plates. Transfection was performed using JetPrime reagent (Polyplus transfection, Illkirch, France) and $1-1.5 \mu \mathrm{g}$ of total IDS plasmid. Experiments were performed $17 \mathrm{~h}$ post-transfections.

\section{Cell lysis and Western blots}

Transfected cells were washed with phosphate-buffered saline (PBS) with $20 \mathrm{mM} \mathrm{N}$-ethylmaleimide (NEM) and were subsequently lysed on ice for $20 \mathrm{~min}$ in $2 \%$ CHAPS (Anatrace, Maumee) in HEPES-buffered saline at $\mathrm{pH} 6.8$ containing $20 \mathrm{mM}$ NEM and protease inhibitor cocktails. After centrifugation at $10,000 \mathrm{~g}$ for $10 \mathrm{~min}$, postnuclear supernatants were collected and aliquoted into Eppendorf tubes. Lysates were reduced and denatured using sample buffer supplemented with dithiothreitol (DTT) for $5 \mathrm{~min}$ at $95^{\circ} \mathrm{C}$. Samples were then separated using $12 \%$ sodium dodecyl sulfate-polyacrylamide gel electrophoresis (SDSPAGE) and were subsequently transferred to polyvinylidene fluoride membrane using Trans-Blot Turbo Transfer System (Bio-Rad, Cressier, Switzerland). Rabbit anti-HA and goat anti-IDS were used as primary antibodies, whereas antiproteinA horseradish peroxidase (HRP) and anti-goat HRP as secondary antibodies. Proteins were revealed with the Luminata Forte Western HRP substrate (Millipore, Schaffhausen, Switzerland). Chemiluminescence was detected by ImageQuant LAS 4000 system in both standard and high acquisition (GE Healthcare Life Sciences, Glattbrugg, Switzerland).

\section{Metabolic labeling, immunoprecipitations, and radiolabeled protein detection}

Proteins produced by HEK cells were radiolabeled for $10 \mathrm{~min}$ using D-MEM containing $0.1 \mathrm{mCi}\left[{ }^{35} \mathrm{~S}\right]$-methionine/ cysteine (pulse solution). Chase was performed in D-MEM supplemented with nonradiolabeled methionine and cysteine. Cells were lysed as described above. Where needed, PS341 or BafA1 was added to the chase solution at concentrations de- scribed above. PNSs were immunoprecipitated using protein $\mathrm{A} /$ protein $\mathrm{G}$ beads and anti-HA/anti-IDS at $4^{\circ} \mathrm{C}$ for $2.5 \mathrm{~h}$. Subsequently, beads were washed twice with $0.5 \%$ CHAPS and proteins were separated in reducing SDS-PAGE. Gels were dried and exposed to autoradiography films (GE Healthcare, Fuji) to reveal radiolabeled bands using Typhoon FLA 9500 software, version 1.0. Protein quantification was performed using ImageQuant software (Molecular Dynamics, GE Healthcare).

\section{Immunofluorescence analyses}

Around $1.5 \times 10^{5}$ MEFs were seeded in 12-well plates on Alcian blue coverslips, transfected as previously described, and incubated with/without $50 \mathrm{nM}$ BafA1. After $12 \mathrm{~h}$, cells were washed twice with PBS and fixed in $3.7 \%$ formaldehyde for $20 \mathrm{~min}$ at room temperature (RT). Then, cells were washed three times with PBS and permeabilized using a solution composed of $10 \%$ goat serum, $15 \mathrm{mM}$ glycine, $0.05 \%$ saponin, and $10 \mathrm{mM}$ HEPES in PBS (PS) for $15 \mathrm{~min}$ at RT. After permeabilization, cells were incubated with the anti-IDS diluted 1:100 and anti-LAMP1 diluted 1:50 in PS for $90 \mathrm{~min}$ at RT. Subsequently, cells were washed three times (5 min each) in PS, supplemented with Alexa Fluor-conjugated secondary antibody diluted 1:300 in PS for $40 \mathrm{~min}$, washed with PS and water, and mounted with Vectashield (Vector Laboratories, Burlingame) containing 4',6-diamidino-2phenylindole (DAPI). Pictures were collected by Leica TCS SP5 microscope with a $63 \times / 1.4$ N.A. objective (Leica HCX PL APO lambda blue $63.0 \times 1.40$ OIL UV, Wetzlar, Germany).

\section{Formylation assessment by mass spectrometry}

After immunoprecipitation, proteins were eluted from the beads by heating beads at $50^{\circ} \mathrm{C}$ for $10 \mathrm{~min}$ in sample buffer containing $2 \%$ SDS (nonreducing conditions). Proteins were then separated by SDS-PAGE on a $12 \%$ acrylamide gel over a distance of $4.0 \mathrm{~cm}$. After rapid Coomassie Blue staining, bands were excised and in-gel reduced, and alkylated with chloroacetamide and digested with chymotrypsin (Promega) as described (Wilm et al., 1996). Data-dependent LC-MS/ MS analyses of extracted peptide mixtures after digestion were carried out on a Fusion tri-hybrid orbitrap mass spectrometer (Thermo Fisher Scientific) interfaced to a Dionex RSLC 3000 nano-HPLC. Peptides were separated on a 60 min gradient from $5 \%$ to $50 \%$ acetonitrile in $0.1 \%$ formic acid at $0.3 \mu \mathrm{L} / \mathrm{min}$ on a PepMap column $(75 \mu \mathrm{m}$ ID $\times 25 \mathrm{~cm}, 2.0 \mu \mathrm{m}, 100 \AA \mu$; Dionex). Full MS survey scans were performed at 120,000 resolution. In data-dependent acquisition controlled by Xcalibur 2.1 software (Thermo Fisher), the 20 most intense multiply charged precursor ions detected in the full MS survey scan were selected for collision-induced dissociation and analysis in the linear trap with an isolation window of $1.6 \mathrm{~m} / \mathrm{z}$, and then dynamically excluded from further selection during $60 \mathrm{~s}$. Collections of tandem mass spectra from all five fractions for each sample were pooled for database searching using Mascot (Matrix Science, London, United Kingdom; version 2.5.0) against the release 2014_08 of the SWISSPROT database restricted to human taxonomy. Mass tolerances used were $10 \mathrm{ppm}$ for the precursors and 0.5 Da for CID fragments. The software Scaffold 4.4.1.1 (Proteome Software, Inc.) was used to validate MS/MS-based peptide identifications (minimum 
95\% probability) (Keller et al., 2002) and protein (minimum 99\% probability) (Nesvizhskii et al., 2003), and perform dataset alignment and subtraction, as well as parsimony analysis to discriminate homologous hits.

\section{IDS enzymatic activity assay}

HEK293 cells were washed twice on ice with PBS without calcium and magnesium. Afterward, $5 \mathrm{mM}$ ethylenediaminetetraacetic acid (EDTA) in PBS was used to gently detach the cells. HEKs were collected in an Eppendorf tube and centrifuged at $1500 \mathrm{~g}$ for $5 \mathrm{~min}$. EDTA solution was removed by washing the pellet twice with PBS. After washing, cells were centrifuged at $1500 \mathrm{~g}$ for $5 \mathrm{~min}$. Enzymatic activity assay was performed by fluorimetric determination of IDS-mediated hydrolyzation of 4-methylumbelliferyl- $\alpha$-L-iduronide-2-sulfate to 4-methylumbelliferone (Voznyi et al., 2001) (Moscerdam, Oegstgeest, Netherlands).

\section{$M D$ simulations}

MD simulations were performed using the X-ray structure of the IDS (PDB ID code 5FQL) (Demydchuk et al., 2017) as a starting point. The five systems used in MD simulations $\left(\mathrm{IDS}_{\mathrm{WT}}, \mathrm{IDS}_{\mathrm{L} 482 \mathrm{X}}, \mathrm{IDS}_{\mathrm{Y} 452 \mathrm{X}}, \mathrm{IDS}_{\mathrm{R} 443 \mathrm{X}}\right.$, and $\left.\mathrm{IDS}_{\mathrm{W} 337 \mathrm{X}}\right)$ were obtained deleting the residue between the truncation site and the C-terminal end of the protein. All systems, consisting of the protein and the covalently bound glycans included in the X-ray structure, were then prepared for MD with "protein preparation wizard module" available in the Schrodinger suite for molecular modeling. This procedure automatically adds missing atoms to side chains, models short unresolved protein loops, and assigns the correct atom names according to the chosen force field. The short loop connecting SD1 to SD2, not solved in the X-ray structure, and susceptible to proteolytic cleavage was modeled in this preparation step. The protonation state of protein residues was assigned using Propka considering a reference $\mathrm{pH}$ of 7.4 (Olsson et al., 2011). The protein structures were then solvated in a box of water with minimal distance from the protein surface of $10 \AA$. The neutrality of the system was ensured adding the proper number of counter ions. All the nonsolvent molecules were described by the OPLS3 (Harder et al., 2016) force field, while TIP3P model (Jorgensen et al., 1983) was used for water molecules. The systems were equilibrated following the equilibration protocol recommended for Desmond MD simulations: (1) Brownian dynamics (100 ps) in an NVT enable ( $T=10 \mathrm{~K})$ applying harmonic restraints on solute heavy atoms (force constant 50 $\mathrm{kcal} / \mathrm{mol} / \AA)$; (2) NVT ( $T=10 \mathrm{~K}) \mathrm{MD}$ simulation of $12 \mathrm{ps}$ in NVT ensemble conserving the same restraints applied in (1); (3) NPT ( $T=300 \mathrm{~K}$ and $P=1 \mathrm{~atm}) \mathrm{MD}$ simulation (12 ps) conserving the same restraints applied in (1); and (4) NPT ( $T=300 \mathrm{~K}$ and $P=1 \mathrm{~atm}) \mathrm{MD}$ simulation (24 ps) without restraints. Finally, all the systems were simulated for $500 \mathrm{~ns}$. The pressure and the temperature were fixed at $300 \mathrm{~K}$ and 1 atm by the Martyna-Tobias-Klein NPT thermostat scheme. Root mean square deviation (RMSD) value were calculated considering only the backbone atoms using the tool implemented in the VMD program (Humphrey et al., 1996). Root mean square fluctuations (RMSF) have been calculated by the g_rmsf tool implemented in GROMACS 2016.5. considering backbone atoms (Abraham et al., 2015).

\section{Results}

\section{MD simulations}

MD simulations allow prediction of protein stability (Adcock and McCammon, 2006; Amir et al., 2019). In our analyses, we compared the structural stability of the 550residue, full-length IDS protein (IDS $\mathrm{WT}_{\mathrm{W}}$, Fig. 1A) with the structural stability of four forms of IDS linked to MPS II and truncated at position $482\left(\operatorname{IDS}_{\mathrm{L} 482 \mathrm{X}}\right), 452$ (IDS $\left._{\mathrm{Y} 452 \mathrm{X}}\right), 443$ $\left(\operatorname{IDS}_{\mathrm{R} 443 \mathrm{X}}\right)$, or 337 (IDS $\left.\mathrm{W}_{\mathrm{W} 37 \mathrm{X}}\right)$, respectively (Fig. 1C). To this end, we monitored structural quantities such as RMSF and RMSD to assess protein stability and deviation from the reference IDS $_{\mathrm{WT}}$ crystal structure (Khan et al., 2016; Demydchuk et al., 2017; Dong et al., 2018). The larger RMSF and RMSD values for $\operatorname{IDS}_{\mathrm{W} 337 \mathrm{X}}$ (Fig. 1D) hint at higher instability of IDS $_{\mathrm{W} 337 \mathrm{X}}$, compared to $\mathrm{IDS}_{\mathrm{WT}}, \mathrm{IDS}_{\mathrm{L} 482 \mathrm{X}}$, IDS $_{\mathrm{Y} 452 \mathrm{X}}$, and $\mathrm{IDS}_{\mathrm{R} 443 \mathrm{X}}$, all characterized by similar RMSF and RMSD values (Fig. 1D). Newly synthesized polypeptides are subjected to a stringent quality control in the ER. Structural instability of $\operatorname{IDS}_{\mathrm{W} 337 \mathrm{X}}$ led us to anticipate the retention of this mutant polypeptide in the ER and its rapid degradation, thus explaining the loss-of-function disease with severe outcome linked to this mutation. What about the other missense mutations for which MD analyses failed to reveal significant stability impairments, but are linked to a loss-of-function disease?

\section{Expression of wild-type and nonsense IDS mutants}

To verify the reliability of the predictions made by MD simulations and to establish the consequences of loss-offunction truncations on IDS maturation and fulfillment of ER quality control requirements for lysosomal transport, we compared the intracellular fate of IDS $_{\mathrm{WT}}$ with that of IDS $_{L_{482 X}}, I_{\text {IDS }} 452 X, I D S_{R 443 X}$, and IDS $_{\mathrm{W} 337 X}$. The five HA-tagged proteins were ectopically expressed in human embryonic kidney cells (HEK293) or in MEF. After detergent solubilization, cellular proteins were separated in SDS-polyacrylamide gels and transferred onto polyvinylidene difluoride membranes (Western blot [WB]). IDS $_{\mathrm{WT}}$ and the four IDS variants were revealed with a polyclonal, IDS-specific antibody (Fig. 2A), or with an anti-HA tag antibody that recognizes the C-terminus of the IDS proteins (Fig. 2B). Consistent with the expected processing pattern (Fig. 1A, B) (Froissart et al., 1995), the wild-type (WT) protein was separated in four distinct bands labeled as FL (full-length, unprocessed precursor), $50 \mathrm{kDa}, \mathrm{SD} 1$ and SD2. These polypeptides are revealed with the IDS-specific antibody (Fig. 2A, lane 1). Only FL was visible in the WB revealed with the anti-HA antibody (Fig. 2B, lane 7). This was expected since the HA-tag displayed at the polypeptide C-terminus is lost when IDS $_{\mathrm{WT}}$ is processed into mature fragments within the lysosome.

IDS $_{\mathrm{L} 482 \mathrm{X}}$ ends 39 residues after the expected processing site, which is at position 443 of the IDS polypeptide. However, the polypeptide runs as a single-polypeptide band when separated by gel electrophoresis (Fig. 2A, lane 2). This reveals that the truncation of most of the SD2 domain impairs proteolytic maturation of IDS $_{\mathrm{L} 482 \mathrm{X}}$ that would generate polypeptides running at the same level as the $50 \mathrm{kDa}$ and the SD1 generated on processing of $\operatorname{IDS}_{\mathrm{WT}}$ (Fig. 2A, lane 1). 

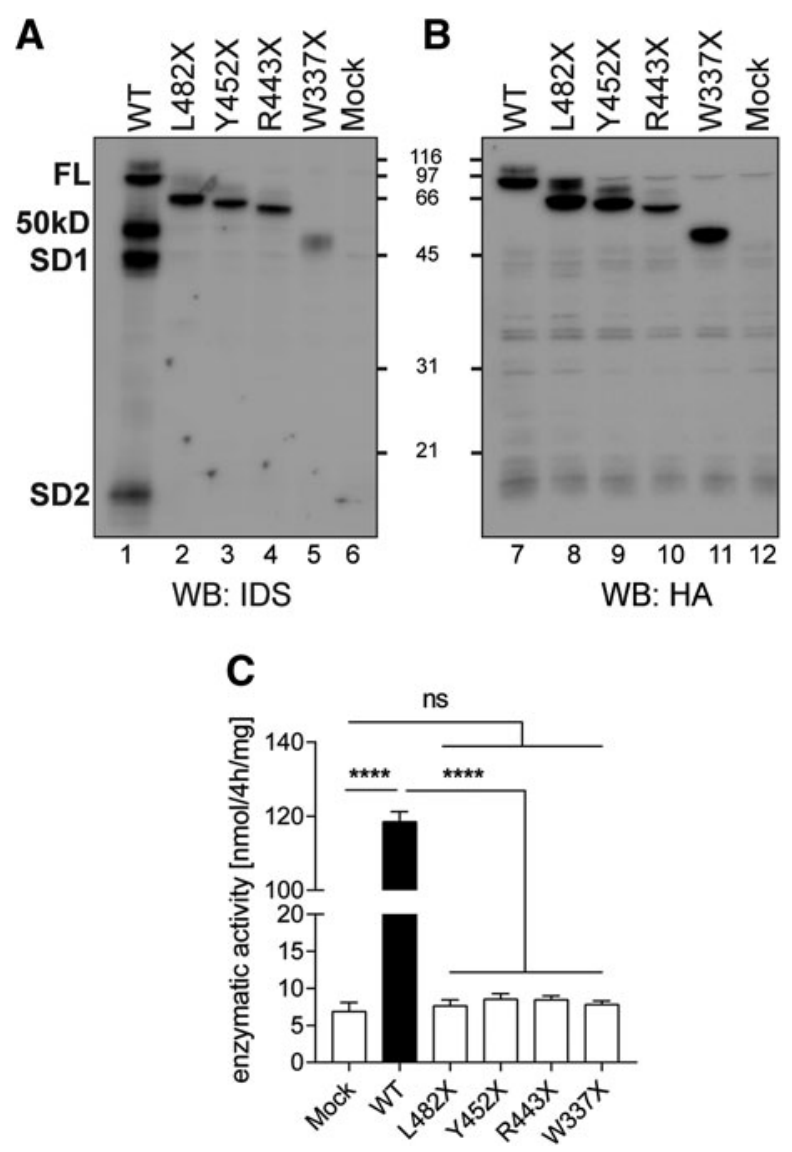

FIG. 2. Ectopic expression of IDS variants and their intracellular activity. (A) $\mathrm{IDS}_{\mathrm{WT}}, \mathrm{IDS}_{\mathrm{L} 482 \mathrm{X}}, \mathrm{IDS}_{\mathrm{Y} 452 \mathrm{X}}$, $\mathrm{IDS}_{\mathrm{R} 443 \mathrm{X}}$, and $\mathrm{IDS}_{\mathrm{W} 337 \mathrm{X}}$ revealed with a polyclonal antibody raised to human IDS. (B) Same as A, where ectopically expressed IDS are revealed with an anti-HA antibody. (C) Fluorimetric determination of IDS-variant hydrolyzation of 4-methylumbelliferyl- $\alpha$-L-iduronide-2-sulfate to 4methylumbelliferone (Voznyi et al., 2001).

The electrophoretic mobility of the $\operatorname{IDS}_{\mathrm{L} 482 \mathrm{X}}$ with an apparent MW of $66 \mathrm{kDa}$ indicates defective processing at the C-terminus (consistently, the polypeptide retains the HA epitope, Fig. 2B, lane 8) and a possible defect in removal of the propeptide (Fig. 1C). The other variants examined in this study are truncated immediately after $\left(\operatorname{IDS}_{\mathrm{Y} 452 \mathrm{X}}\right)$, or at the physiologic site of IDS cleavage $\left(\operatorname{IDS}_{\mathrm{R} 443 \mathrm{X}}\right)$, respectively (Fig. 1C). They have an apparent MW of 60 and $55 \mathrm{kDa}$ (Fig. 2A, lanes 3 and 4, respectively), which is consistent with the presence of the HA tag (confirmed in Fig. 2B, lanes 9 and 10, respectively) and a possible defect in removal of the propeptide (Fig. 1C). IDS $_{\mathrm{W} 337 \mathrm{X}}$ conserves all $\mathrm{N}$-glycans of the SD1 domain, but lacks $25 \%$ of the amino acid stretch and one of the two intramolecular disulfide bonds (Fig. 1C). It runs with an apparent $\mathrm{MW}$ of $47 \mathrm{kDa}$; it is poorly recognized by the anti-IDS polyclonal antibody (Fig. 2B, lane $5)$, but is very efficiently detected by the anti-HA antibody (Fig. 2B, lane 11). All in all, analyses of total cell lysates revealed high-level expression of all IDS variants examined in this study. Based on the WB analyses, we can speculate that only IDS $_{\mathrm{WT}}$ is appropriately processed within lysosomes into the mature form.

\section{Activity measurement and formylation of IDS variants}

Oxidation of the thiol group of Cys84 in the Cys-Ala-ProSer-Arg- sequon to 2-formylglycine by formylglycinegenerating enzyme (Dierks et al., 1999) is required for activity of eukaryotic sulfatases and occurs in the ER either cotranslationally or post-translationally (Schmidt et al., 1995). Mass spectrometry of chymotryptic fragments of IDS $_{\mathrm{WT}}$, $\mathrm{IDS}_{\mathrm{L} 482 \mathrm{X}}, \mathrm{IDS}_{\mathrm{Y} 452 \mathrm{X}}, \mathrm{IDS}_{\mathrm{R} 443 \mathrm{X}}$, and $\mathrm{IDS}_{\mathrm{W} 337 \mathrm{X}}$ (see Materials and Methods section) revealed that all proteins were appropriately modified at cysteine 84 . However, when the enzymatic activity was monitored by fluorimetric determination of the IDS-mediated hydrolyzation of 4-methylumbelliferyl$\alpha$-L-iduronide-2-sulfate to 4-methylumbelliferone according to a well-established diagnostic assay (Voznyi et al., 2001), only cells expressing IDS $_{\mathrm{WT}}$ were considered competent (Fig. 2C). Lack of enzymatic activity for cells expressing IDS $_{\mathrm{W} 337 \mathrm{X}}$ was not surprising. IDS $_{\mathrm{W} 337 \mathrm{X}}$ is linked to severe MPS II; it lacks $25 \%$ of the catalytic domain, including one catalytic residue (Lys347) and the $\mathrm{Cys}_{422}-\mathrm{S}-\mathrm{S}-\mathrm{Cys}_{432}$ disulfide bond, which is crucial for enzymatic activity. Moreover, our MD analyses predict extensive instability of this polypeptide (Fig. 1D). However, enzymatic activity was also undetectable in cells expressing the $\mathrm{IDS}_{\mathrm{L} 482 \mathrm{X}}$, ID$\mathrm{S}_{\mathrm{Y} 452 \mathrm{X}}$, and $\operatorname{IDS}_{\mathrm{R} 443 \mathrm{X}}$ (Fig. 2C), for which MD analyses failed to reveal structural instability and which contain the entire catalytic domain with the 10 active site residues that are highly conserved among sulfatases (D45, D46, C84, R88, K135, H138, H229, D334, H335, and K347) (Demydchuk et al., 2017). Lack of enzymatic activity is consistent with our speculation based on Figure 2A and B that only IDS $_{\mathrm{WT}}$ is appropriately processed within lysosomes into the mature form.

\section{Time-resolved analyses of IDS fate}

To determine the fate of newly synthesized IDS variants, cells expressing the model polypeptides were pulse labeled with ${ }^{35} \mathrm{~S}$-methionine and cysteine for $10 \mathrm{~min}$. Radiolabeled IDS was immunoisolated from cell lysates at increasing times after the pulse (10-360 min, Fig. 3A-E). Immunoisolation of radiolabeled IDS variants with the anti-HA that recognizes the unprocessed forms of the proteins revealed that in all cases, these forms disappeared during the chase (Fig. 3A-E, lanes 1-6). The immunoisolation of the radiolabeled IDS variants with an anti-IDS antibody revealed that disappearance of the HA epitope correlated with disappearance of the IDS signal for IDS $_{\mathrm{L} 482 \mathrm{X}}$ (Fig. 3B, lanes 7-12), IDS $_{\mathrm{Y} 452 \mathrm{X}}$

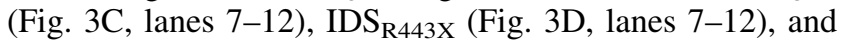
IDS $_{\text {W337X }}$ (Fig. 3E, lanes 7-12) variants, showing that these proteins were degraded during the chase. In contrast, for the WT protein, disappearance of the HA epitope (Fig. 3A, lanes 1-6) resulted from processing of the polypeptide Cterminus as indicated by the appearance of proteolytic fragments recognized by the anti-IDS antibody (Fig. 3A, arrows in lanes 10-12) corresponding to the $50 \mathrm{kDa}$ and the SD1 proteolytic fragments shown by WB (Fig. 2A, lane 1). These results show that the truncated variants of IDS were degraded during the chase, whereas the WT form was proteolytically processed within the lysosomes in the enzymatically active form of IDS, and are consistent with the finding reported above that the IDS activity is only measured in cells expressing the WT form of the protein. 


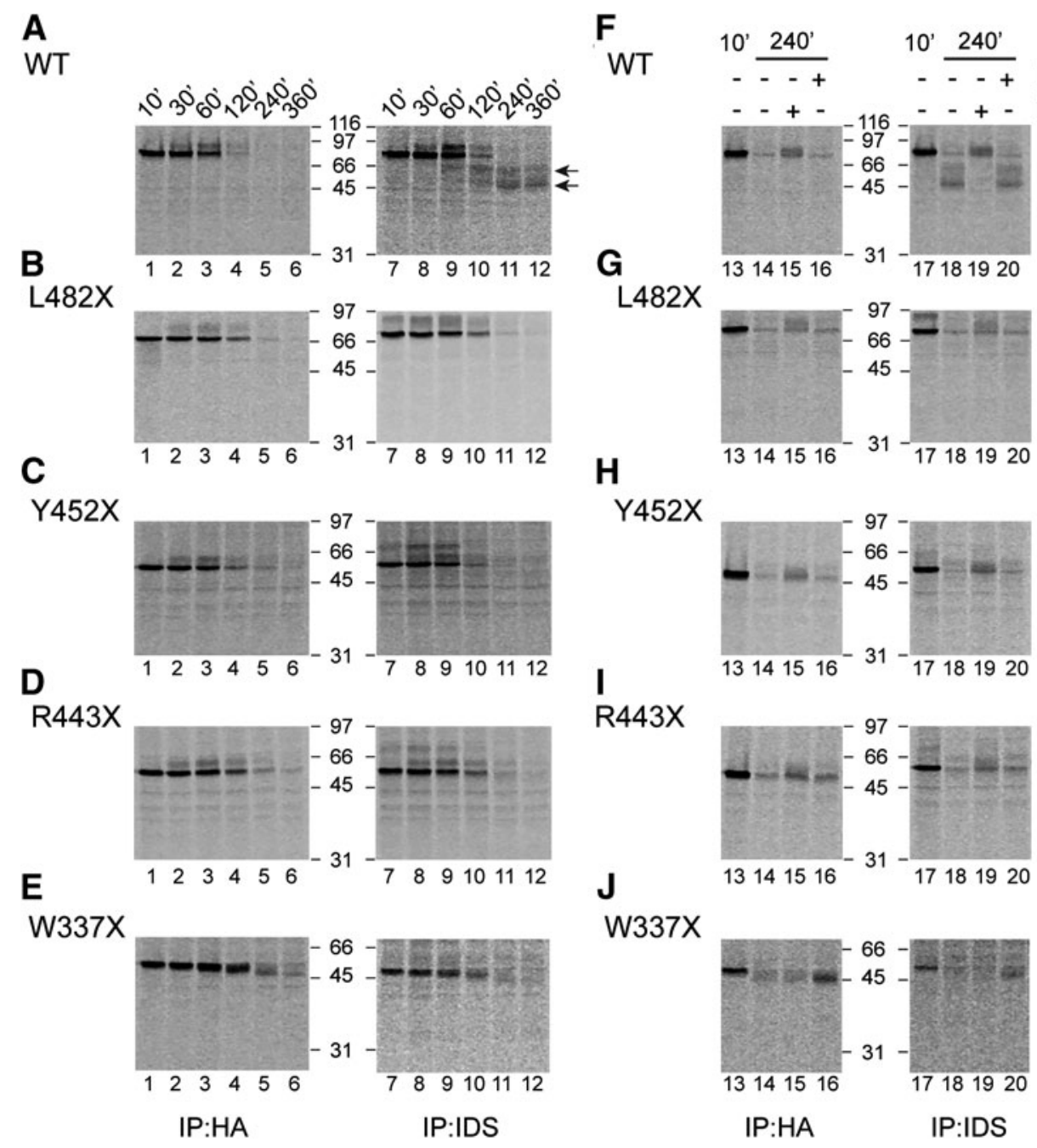

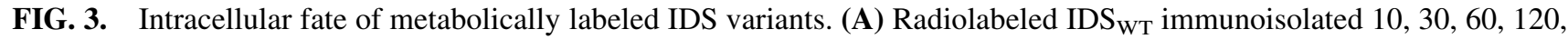
240, and 360 min after synthesis with anti-HA antibodies (lanes 1-6) or with an anti-IDS antibody (lanes 7-12). (B) Same as

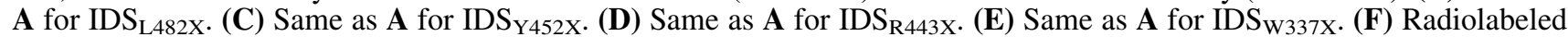
IDS $_{\mathrm{WT}}$ immunoisolated 10 min after synthesis (lane 13 with anti-HA and lane 17 with anti-IDS) or 240 min after synthesis from untreated cells (lanes 14 and 18), from cells exposed to the lysosomal inhibitor BafA1 (lanes 15 and 19), or to the proteasomal inhibitor PS341 (lanes 16 and 20). (G) Same as $\mathbf{F}$ for $\operatorname{IDS}_{\mathrm{L} 482 \mathrm{X}}$. (H) Same as $\mathbf{F}$ for IDS $\mathrm{I}_{\mathrm{Y} 452 \mathrm{X}}$. (I) Same as $\mathbf{F}$ for $\mathrm{IDS}_{\mathrm{R} 443 \mathrm{X}}$. (J) Same as $\mathbf{F}$ for $\operatorname{IDS}_{\mathrm{W} 337 \mathrm{X}}$.

\section{Lysosomal and proteasomal degradation of truncated IDS variants}

Protein folding is error prone and the efficiency of the folding process is reduced by mutations in the polypeptide chains. Most folding-defective polypeptides produced in the ER are eventually dislocated into the cytosol for proteasomal degradation in processes defined as ERAD. In the particular case of Hunter's syndrome, ERAD has been proposed as the catabolic pathway that clears from cells mutant forms of IDS, thus causing the loss-of-function phenotype characterizing the disorder (Osaki et al., 2018, 2019). To verify the involvement of ERAD in disposal of the truncated versions of IDS analyzed in our work, cells expressing IDS $_{\mathrm{WT}}, \mathrm{IDS}_{\mathrm{L} 482 \mathrm{X}}, \mathrm{IDS}_{\mathrm{Y} 452 \mathrm{X}}, \mathrm{IDS}_{\mathrm{R} 443}$, and IDS $_{\mathrm{W} 337 \mathrm{X}}$ were metabolically labeled for $10 \mathrm{~min}$ and chased for $10 \mathrm{~min}$ to visualize the initial amount of IDS expressed in the cells (Fig. 3F-J, lane 13 for HA-tagged IDS and lane 17 for total IDS) or for $240 \mathrm{~min}$ to appreciate the disappearance of HA-tagged IDS (Fig. 3F-J, lane 14), or of the total IDS signal (lane 18) during the chase. The 240-min chase time was also performed for cells grown in the presence of an inhibitor of lysosomal (BafA1, Fig. 3F-J, lanes 15 and 19) or proteasomal activity (PS341, lanes 16 and 20). For IDS $\mathrm{WT}_{\mathrm{WT}}$, as shown in Fig. 3A, lanes 1-6, the HA-epitope disappears during the chase (Fig. 3F, lane 13-14). IDS ${ }_{W T}$ is in fact processed on delivery within lysosomes to the enzymatically active form (Fig. 3A, lanes 7-12 and 3F, lanes 17-18) (Froissart et al., 1995). Proteasomal inactivation does not affect disappearance of the HA epitope (Fig. 3F, lane 16) or the processing of $\operatorname{IDS}_{\mathrm{WT}}$ (lane 20). In contrast, lysosomal inactivation inhibits both disappearance of the HA epitope (Fig. 3F, lane 15) and the formation of the processed forms of IDS $_{\mathrm{WT}}$ (lane 19). This is consistent with 
the fact that IDS $\mathrm{S}_{\mathrm{WT}}$ is delivered within lysosomes, where it is proteolytically cleaved in the active form of the protein (Fig. 1B) (Froissart et al., 1995). For the truncated variants $\mathrm{IDS}_{\mathrm{L} 482 \mathrm{X}}, \mathrm{IDS}_{\mathrm{Y} 452 \mathrm{X}}$, and $\mathrm{IDS}_{\mathrm{R} 443 \mathrm{X}}$ that disappear during the chase without forming distinguishable proteolytic fragments
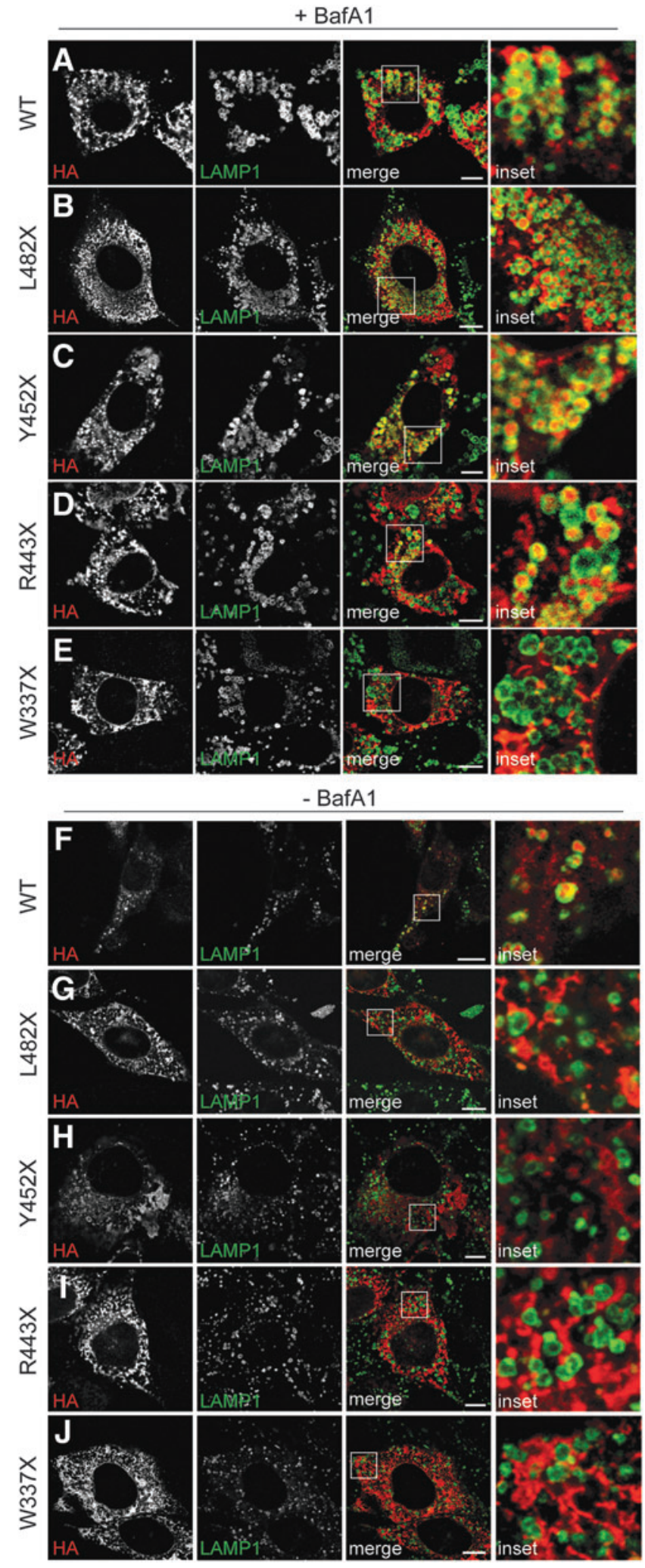

(Fig. 3B-D), proteasomal inactivation does not significantly affect clearance (Fig. 3G-I, lane 16 and 20), whereas lysosomal inactivation stabilizes the proteins (lanes 15, 19). In sharp contrast, clearance from cells of the shortest variant of IDS analyzed in this study (IDS ${ }_{\mathrm{W} 337 \mathrm{x}}$ ) is inhibited on proteasomal (Fig. 3J, lanes 16, 20), but not on lysosomal, inactivation (lanes 15, 19). All in all, IDS ${ }_{\mathrm{WT}}$ is delivered within lysosomes, where it is activated on proteolytic processing. IDS $_{\mathrm{L} 482 \mathrm{X}}, \mathrm{IDS}_{\mathrm{Y} 452 \mathrm{X}}$, and $\mathrm{IDS}_{\mathrm{R} 443 \mathrm{X}}$ pass ER quality control and are eventually delivered within lysosomes, where they undergo proteolytic degradation. Consistent with MD analyses that highlighted the IDS $_{\mathrm{W} 337 \mathrm{X}}$ as the most perturbed form of the protein, this aberrant polypeptide remains trapped by the ER quality control machinery, which selects it for ERAD.

\section{Lysosomal delivery of WT and truncated IDS variants}

We reasoned that if our conclusions were correct, confocal laser scanning microscopy (CLSM) should reveal lysosomal localization of IDS $\mathrm{WT}_{\mathrm{WT}}$ at steady state. In contrast, $\mathrm{IDS}_{\mathrm{L} 482 \mathrm{X}}, \mathrm{IDS}_{\mathrm{Y} 452 \mathrm{X}}$, and $\mathrm{IDS}_{\mathrm{R} 443 \mathrm{X}}$ that are degraded by lysosomal enzymes, should be visible within lysosomes only upon lysosomal inactivation. Lysosomal inactivation should not result in accumulation of IDS $_{\mathrm{W} 337 \mathrm{X}}$ within lysosomes, as this IDS variant is an ERAD substrate. To verify this, cells expressing $\mathrm{IDS}_{\mathrm{WT}}, \mathrm{IDS}_{\mathrm{L} 482 \mathrm{X}}, \mathrm{IDS}_{\mathrm{Y} 452 \mathrm{X}}, \mathrm{IDS}_{\mathrm{R} 443 \mathrm{X}}$, and IDS $_{\mathrm{W} 337 \mathrm{X}}$ were incubated for $12 \mathrm{~h}$ with BafA1 before processing for CLSM. As predicted, in these cells, all IDS variants (Fig. 4A-D), but $\operatorname{IDS}_{\mathrm{W} 337 \mathrm{X}}$ (Fig. 4E), did accumulate in lysosomes displaying LAMP1 at their limiting membrane. On BafA1 washout, only IDS $_{\mathrm{WT}}$ was stable in the lysosomes (Fig. 4F), where it is proteolytically processed to the mature form (Fig. 2A, lane 1; Fig. 3A, lanes 712; Fig. 3F, lanes 17-19) and acquires enzymatic activity (Fig. 2C). $\operatorname{IDS}_{\mathrm{L} 482 \mathrm{X}}, \mathrm{IDS}_{\mathrm{Y} 452 \mathrm{X}}$, and $\mathrm{IDS}_{\mathrm{R} 443 \mathrm{X}}$ disappeared from the lysosomal lumen consistent with their intracellular degradation (Fig. 3B-D), which is executed in the lysosomes (Fig. 3G-I, lanes 15 and 19). IDS $_{\mathrm{W} 337 \mathrm{X}}$, which is linked to the most severe form of MPS II, is dramatically misfolded (Fig. 1D), does not pass ER quality control, and is translocated in the cytosol for proteasomal degradation (Fig. 3J, lanes 16 and 20).

\section{Discussion}

Our analyses of the intracellular fate of IDS $_{\mathrm{WT}}$ and of 4 disease-linked truncated versions of IDS reveal a correlation between structural instability as predicted by MD

FIG. 4. Delivery of IDS variants within LAMP1-positive lysosomes and their stability. (A) CLSM showing the intracellular localization of ectopically expressed IDS $\mathrm{WT}_{\mathrm{WT}}$ (anti-HA immunoreactivity, red) and its delivery within LAMP1positive lysosomes (green). Cells have been exposed to the lysosomal inhibitor BafA1 to preserve the IDS variants that are delivered within the degradative compartment. (B) Same as $\mathbf{A}$ for $\operatorname{IDS}_{\mathrm{L} 482 \mathrm{X}}$. (C) Same as $\mathbf{A}$ for IDS $_{\mathrm{Y} 452 \mathrm{X}}$. (D) Same as $\mathbf{A}$ for $\operatorname{IDS}_{\mathrm{R} 443 \mathrm{X}}$. (E) Same as $\mathbf{A}$ for IDS $_{\mathrm{W} 337 \mathrm{X}}$ ( $\left.\mathbf{F}-\mathbf{J}\right)$ Same as A-E in the absence of BafA1. Scale bars $10 \mu \mathrm{m}$. CLSM, confocal laser scanning microscopy. 
calculations and failure to pass ER quality control checkpoints for the shortest IDS variant under investigation. IDS $_{\mathrm{W} 337 \mathrm{X}}$, which is linked to severe forms of MPS II, is retained in the ER and is dislocated across the ER membrane for proteasomal degradation. As such, $\operatorname{IDS}_{\mathrm{W} 337 \mathrm{X}}$ is a classical substrate of the ERAD machinery, whose failure to attain a native structure prevents transport at the organelle of destination, in this case the lysosome, and results in a lossof-function LSD. The other truncated versions of IDS analyzed in our study are not distinguished from the WT form of the protein by MD analyses. They all pass ER quality control to some extent and are appropriately transported within lysosomes. In sharp contrast to IDS $_{\mathrm{WT}}$, however, truncated forms of IDS are rapidly degraded in the lysosomal lumen and their activity remains below detection, thereby explaining the loss-of-function disease state of the carriers of these nonsense mutations. The ER is site of production of all secretory and membrane-bound proteins and of proteins destined to the cellular organelles, including the lysosome. It is considered the site where the gene products are subjected to a stringent quality control that selects structurally imperfect gene products for degradation, thus resulting in loss-of-function diseases. As such, modulation of processes occurring within the ER such as posttranslational modifications, chaperone-assisted protein folding, selection for secretion, or for degradation are strong candidates for therapeutic intervention, and chemical and pharmacologic chaperones that enhance folding of mutant gene products are entering the clinics (Noack et al., 2014; Pilla et al., 2017). Our data reveal that passing ER quality control and arrival at the intracellular site of destination might not be sufficient to warrant healthy status. They highlight lysosomal quality control as the distal station to control lysosomal enzyme fitness and pave the way for alternative therapeutic interventions.

\section{Acknowledgments}

We thank Fatima and Mike, the members of Molinari's laboratory for discussions and M. Quadroni (Protein Analysis Facility of the University of Lausanne) for mass spectrometry (MS) analyses.

\section{Disclosure Statement}

No competing financial interests exist.

\section{Funding Information}

M.M. is supported by Signora Alessandra, AlphaONE Foundation, Foundation for Research on Neurodegenerative Diseases, Swiss National Science Foundation, and Comel and Gelu Foundations.

\section{References}

Abraham, M.J., Murtola, T., Schulz, R., Páll, S., Smith, J.C., Hess, B., et al. (2015). GROMACS: high performance molecular simulations through multi-level parallelism from laptops to supercomputers. SoftwareX 1-2, 19-25.

Adcock, S.A., and McCammon, J.A. (2006). Molecular dynamics: survey of methods for simulating the activity of proteins. Chem Rev 106, 1589-1615.
Amir, M., Mohammad, T., Kumar, V., Alajmi, M.F., Rehman, M.T., Hussain, A., et al. (2019). Structural analysis and conformational dynamics of STN1 gene mutations involved in coat plus syndrome. Front Mol Biosci 6, 41.

Bach, G., Eisenberg, F., Jr., Cantz, M., and Neufeld, E.F. (1973). The defect in the Hunter syndrome: deficiency of sulfoiduronate sulfatase. Proc Natl Acad Sci U S A 70, 21342138.

Brusius-Facchin, A.C., Schwartz, I.V., Zimmer, C., Ribeiro, M.G., Acosta, A.X., Horovitz, D., et al. (2014). Mucopolysaccharidosis type II: identification of 30 novel mutations among Latin American patients. Mol Genet Metab 111, 133 138.

Demydchuk, M., Hill, C.H., Zhou, A., Bunkoczi, G., Stein, P.E., Marchesan, D., et al. (2017). Insights into Hunter syndrome from the structure of iduronate-2-sulfatase. Nat Commun $\mathbf{8}$, 15786.

Dierks, T., Lecca, M.R., Schlotterhose, P., Schmidt, B., and von Figura, K. (1999). Sequence determinants directing conversion of cysteine to formylglycine in eukaryotic sulfatases. EMBO J 18, 2084-2091.

Dong, Y.W., Liao, M.L., Meng, X.L., and Somero, G.N. (2018). Structural flexibility and protein adaptation to temperature: molecular dynamics analysis of malate dehydrogenases of marine molluscs. Proc Natl Acad Sci U S A 115, 1274-1279.

Filocamo, M., Bonuccelli, G., Corsolini, F., Mazzotti, R., Cusano, R., and Gatti, R. (2001). Molecular analysis of 40 Italian patients with mucopolysaccharidosis type II: new mutations in the iduronate-2-sulfatase (IDS) gene. Hum Mutat 18, 164-165.

Froissart, R., Maire, I., Millat, G., Cudry, S., Birot, A.M., Bonnet, V., et al. (1998). Identification of iduronate sulfatase gene alterations in 70 unrelated Hunter patients. Clin Genet 53, 362-368.

Froissart, R., Millat, G., Mathieu, M., Bozon, D., and Maire, I. (1995). Processing of iduronate 2-sulphatase in human fibroblasts. Biochem J 309 (Pt 2), 425-430.

Harder, E., Damm, W., Maple, J., Wu, C., Reboul, M., Xiang, J.Y., et al. (2016). OPLS3: a force field providing broad coverage of drug-like small molecules and proteins. J Chem Theory Comput 12, 281-296.

Humphrey, W., Dalke, A., and Schulten, K. (1996). VMD: visual molecular dynamics. J Mol Graph 14, 33-38.

Hunter, C. (1917). A rare disease in two brothers. Proc R Soc Med 10, 104-116.

Jorgensen, W.L., Chandrasekhar, J., Madura, J.D., Impey, R.W., and Klein, L.M. (1983). Comparison of simple potential functions for simulating liquid water. J Chem Phys 79, 926-935.

Keeratichamroen, S., Cairns, J.R., Wattanasirichaigoon, D., Wasant, P., Ngiwsara, L., Suwannarat, P., et al. (2008). Molecular analysis of the iduronate-2-sulfatase gene in Thai patients with Hunter syndrome. J Inherit Metab Dis 31 Suppl 2, S303-S311.

Keller, A., Nesvizhskii, A.I., Kolker, E., and Aebersold, R. (2002). Empirical statistical model to estimate the accuracy of peptide identifications made by MS/MS and database search. Anal Chem 74, 5383-5392.

Khan, S., Farooq, U., and Kurnikova, M. (2016). Exploring protein stability by comparative molecular dynamics simulations of homologous hyperthermophilic, mesophilic, and psychrophilic proteins. J Chem Inf Model 56, 2129-2139.

Kosuga, M., Mashima, R., Hirakiyama, A., Fuji, N., Kumagai, T., Seo, J.H., et al. (2016). Molecular diagnosis of 65 families 
with mucopolysaccharidosis type II (Hunter syndrome) characterized by 16 novel mutations in the IDS gene: genetic, pathological, and structural studies on iduronate-2-sulfatase. Mol Genet Metab 118, 190-197.

Marques, A.R.A., and Saftig, P. (2019). Lysosomal storage disorders-challenges, concepts and avenues for therapy: beyond rare diseases. J Cell Sci 132, jcs221739.

Muenzer, J., Beck, M., Eng, C.M., Escolar, M.L., Giugliani, R., Guffon, N.H., et al. (2009). Multidisciplinary management of Hunter syndrome. Pediatrics 124, e1228-e1239.

Nesvizhskii, A.I., Keller, A., Kolker, E., and Aebersold, R. (2003). A statistical model for identifying proteins by tandem mass spectrometry. Anal Chem 75, 4646-4658.

Noack, J., Brambilla Pisoni, G., and Molinari, M. (2014). Proteostasis: bad news and good news from the endoplasmic reticulum. Swiss Med Wkly 144, w14001.

Olsson, M.H., Sondergaard, C.R., Rostkowski, M., and Jensen, J.H. (2011). PROPKA3: consistent treatment of internal and surface residues in empirical $\mathrm{pKa}$ predictions. J Chem Theory Comput 7, 525-537.

Osaki, Y., Matsuhisa, K., Che, W., Kaneko, M., Asada, R., Masaki, T., et al. (2019). Calnexin promotes the folding of mutant iduronate 2-sulfatase related to mucopolysaccharidosis type II. Biochem Biophys Res Commun 514, 217-223.

Osaki, Y., Saito, A., Kanemoto, S., Kaneko, M., Matsuhisa, K., Asada, R., et al. (2018). Shutdown of ER-associated degradation pathway rescues functions of mutant iduronate 2-sulfatase linked to mucopolysaccharidosis type II. Cell Death Dis 9, 808.

Pilla, E., Schneider, K., and Bertolotti, A. (2017). Coping with protein quality control failure. Annu Rev Cell Dev Biol 33, 439-465.

Schmidt, B., Selmer, T., Ingendoh, A., and Vonfigura, K. (1995). A novel amino-acid modification in sulfatases that is defective in multiple sulfatase deficiency. Cell 82, 271-278.

Sohn, Y.B., Ki, C.S., Kim, C.H., Ko, A.R., Yook, Y.J., Lee, S.J., et al. (2012). Identification of 11 novel mutations in 49 Korean patients with mucopolysaccharidosis type II. Clin Genet 81, 185-190.

Stenson, P.D., Mort, M., Ball, E.V., Shaw, K., Phillips, A., and Cooper, D.N. (2014). The human gene mutation database: building a comprehensive mutation repository for clinical and molecular genetics, diagnostic testing and personalized genomic medicine. Hum Genet 133, 1-9.

Sukegawa, K., Tomatsu, S., Fukao, T., Iwata, H., Song, X.Q., Yamada, Y., et al. (1995). Mucopolysaccharidosis type-II (Hunter-disease)-identification and characterization of 8point mutations in the iduronate-2-sulfatase gene in Japanese patients. Hum Mutat 6, 136-143.

Ulloa-Aguirre, A., Janovick, J.A., Brothers, S.P., and Conn, P.M. (2004). Pharmacologic rescue of conformationallydefective proteins: implications for the treatment of human disease. Traffic 5, 821-837.

Vafiadaki, E., Cooper, A., Heptinstall, L.E., Hatton, C.E., Thornley, M., and Wraith, J.E. (1998). Mutation analysis in 57 unrelated patients with MPS II (Hunter's disease). Arch Dis Child 79, 237-241.

Voznyi, Y.V., Keulemans, J.L., and van Diggelen, O.P. (2001). A fluorimetric enzyme assay for the diagnosis of MPS II (Hunter disease). J Inherit Metab Dis 24, 675-680.

Whiteman, D.A.H., and Kimura, A. (2017). Development of idursulfase therapy for mucopolysaccharidosis type II (Hunter syndrome): the past, the present and the future. Drug Des Devel Ther 11, 2467-2480.

Wilm, M., Shevchenko, A., Houthaeve, T., Breit, S., Schweigerer, L., Fotsis, T., et al. (1996). Femtomole sequencing of proteins from polyacrylamide gels by nano-electrospray mass spectrometry. Nature 379, 466-469.

Wraith, J.E., Scarpa, M., Beck, M., Bodamer, O.A., De Meirleir, L., Guffon, N., et al. (2008). Mucopolysaccharidosis type II (Hunter syndrome): a clinical review and recommendations for treatment in the era of enzyme replacement therapy. Eur J Pediatr 167, 267-277.

\section{Address correspondence to: Maurizio Molinari, PhD Institute for Research in Biomedicine Bellinzona $\mathrm{CH}-6500$ \\ Switzerland \\ E-mail: maurizio.molinari@irb.usi.ch}

Received for publication November 18, 2019; accepted November 19, 2019. 